\title{
Bringing Cuban Biotech Research to Bear on COVID-19: All Hands and Minds on Deck
}

\section{Lila Castellanos-Serra PhD DSc}

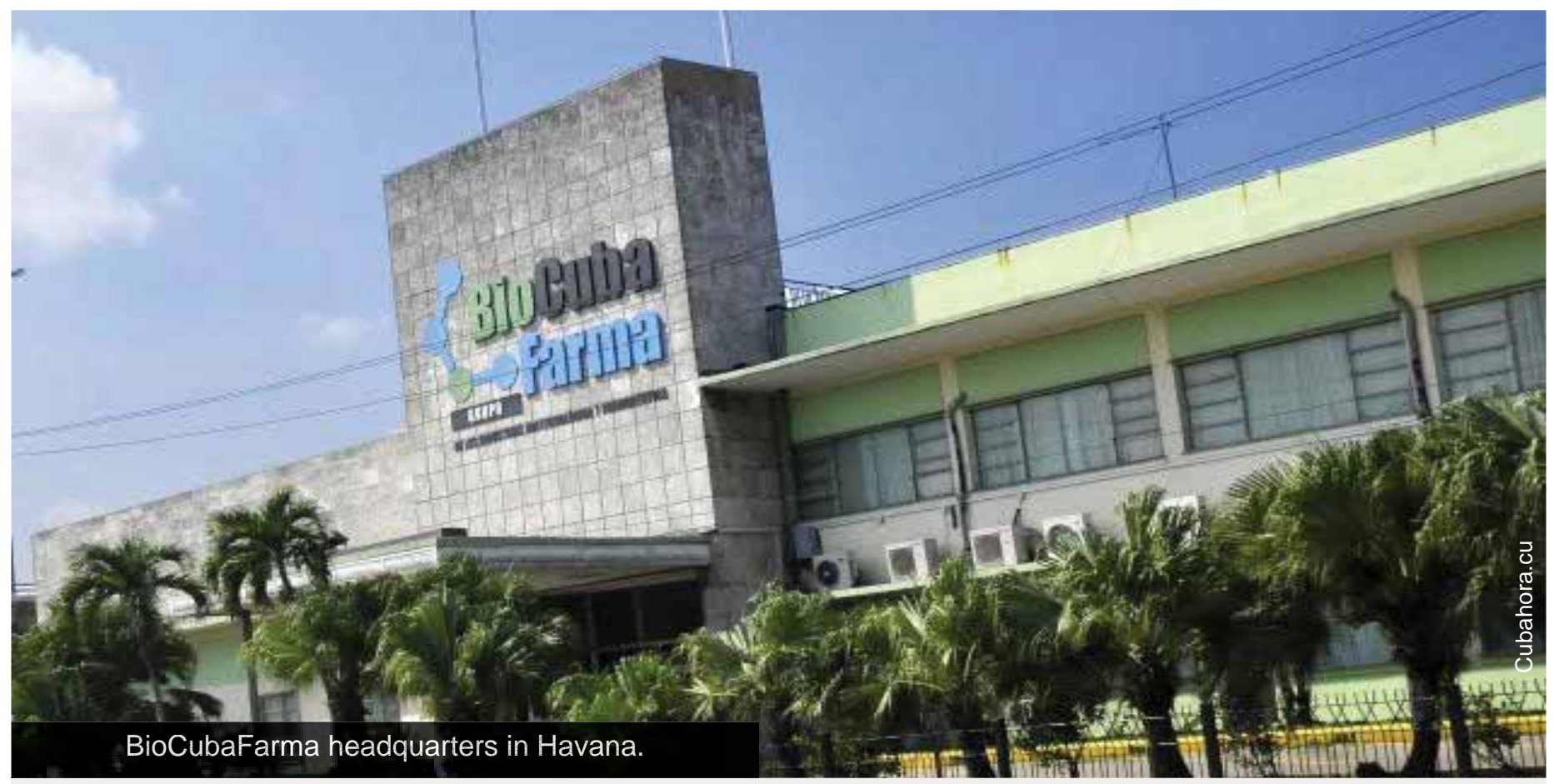

This MEDICC Review roundtable gathers some of Cuba's top researchers in the fields of vaccines and biotechnology, all of whom work in institutions belonging to BioCubaFarma, the umbrella company of Cuban biotech and pharmaceutical R\&D, production, distribution and export. Founded in 2012, the company is comprised of 34 enterprises with 61 lines of production and some 20,000 employees. A total of 765 of its products are registered in 53 countries and exported to another 50. Its scientists' research has resulted in 2640 patents in Cuba and globally.

\section{Introducing our readers to roundtable participants (in order of appearance):}

Eduardo Martínez-Díaz PhD is a biologist with a doctorate in biological sciences and is president of BioCubaFarma since 2017. Prior to that, he served as the company's vice president as well as director of scientific policy. Earlier, he served as vice director of the Genetic Engineering and Biotechnology Center (CIGB) and director of technological development at CIGB.

Vicente Vérez-Bencomo PhD is a chemist with a doctorate in chemical sciences and directs the Finlay Vaccine Institute. He is also founding director of the Synthetic Antigen Laboratory at the University of Havana, where he led the team that discovered and developed the Cuban Haemophilus influenzae type b (Hib) vaccine using a synthetic antigen - the first of its kind in the world.

María del Carmen Domínguez-Horta PhD is a biochemist with a doctorate in biological sciences. She is a senior researcher at CIGB, where she is director of projects in molecular biology, immunodiagnostics and immunology, carrying out clinical trials in search of new drugs for treatment of autoimmune diseases, based on induction of peripheral tolerance.
Rolando Pérez-Rodríguez PhD is a physicist with a doctorate in biological sciences. He is a founder of the Molecular Immunology Center (CIM) and served as its director of research. He is director of science and innovation at BioCubaFarma.

Luis Herrera-Martínez MD PhD is a physician with a doctorate in biological sciences. His team is credited with developing Cuba's first recombinant biotech products, among them, recombinant interferon alfa-2b in 1986. He is a founder of the Center for Biological Research (1982) and of CIGB (1986). He served as research director at both institutions. At CIGB, he was also director of production and technological development and later director. He is currently advisor to the president of BioCubaFarma.

Gerardo Guillén-Nieto PhD is a chemist with a doctorate in biological sciences. Since 1998, he has served as director of biomedical research at CIGB, with main areas of study concerning development of new vaccines. 


\section{MEDICC Review: What do we know about the behavior of COVID-19 and the SARS-CoV-2 virus that causes it?}

Eduardo Martínez: As of April 17, 2020, reported case fatality worldwide is $6.86 \%$; in some countries it's as high as $15 \%$. About $80 \%$ of people infected remain asymptomatic or are only mildly ill, while $20 \%$ become seriously or critically ill. In Cuba thus far, $9 \%$ have become seriously ill and $4 \%$ critically ill, with a case fatality of $3.3 \%$. In these severely ill patients, viral load is much higher than in patients with mild disease, and once the virus establishes itself, it replicates rapidly and the immune system is unable to stop it.

Vicente Vérez: What happens to someone once they're infected depends on the viral load. The higher the viral load, the more difficult it is for the immune system to fight the disease. Many people manage to achieve equilibrium between virus and host: the virus doesn't win, but neither does the host succeed in eliminating it. These people are asymptomatic, and neither they nor the health system know that they are infected unless there is mass population screening. They are the bane of health systems and the main source of transmission.

In individual terms, however, these people are successful because their immune systems have protected them from the virus. We need to understand how some people manage to remain asymptomatic, what characterizes their immune systems that provides such protection and avoids disease development. This is a research question being tackled worldwide.

When that equilibrium between virus and host is broken, when the virus manages to overcome the immune system's defenses, infected people move on to disease states ranging from mild to critical. The virus manipulates the immune system and provokes responses that can be very harmful. Most people who die during the transition to serious or critical illness do not do so directly from the virus: strictly speaking, it's not the virus that kills them, but an intense immune response to the virus. An immune overresponse, called a cytokine storm, attacks the host, finally leading to multisystem organ failure, the nightmare of all intensive care specialists.

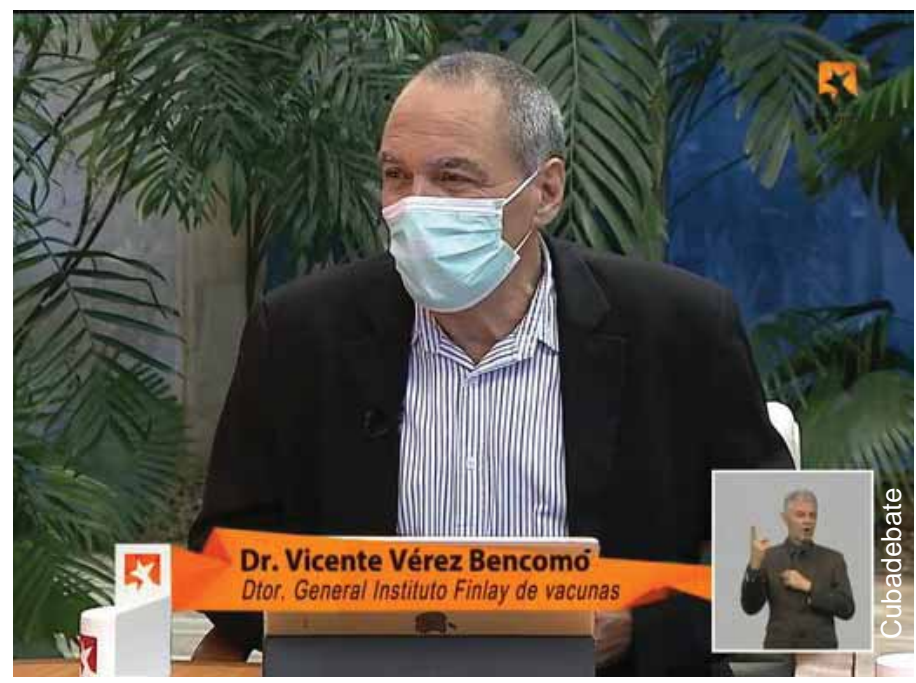

Dr Vicente Vérez appears on Cuba's prime-time Mesa Redonda.
María del Carmen Domínguez: Reports of cytokine storm in COVID-19 patients started to emerge in mid-March this year, the first from a group of Chinese colleagues who described what happens immunologically in a group of patients in transition to critical condition. They develop this hyperinflammatory process, the cytokine storm. Inflammation is a normal physiologic process that helps the body's defenses against a pathogen, such as a virus. But when inflammation is so acute, an excess of cytokines ends up damaging the body. The patients develop respiratory problems requiring artificial ventilation, while thromboses emerge in other organs.

MEDICC Review: While we wait for a preventive vaccine, which will take some time, how can we help the immune system better fight the virus?

Vicente Vérez: There are two kinds of immunity, one specific to a particular disease (usually achieved through a preventive vaccine derived from an inactivated component of the virus) and the other nonspecific. In the case of SARS-CoV-2: when we find equilibrium between virus and host, it is the nonspecific immunity that is responsible for this, and thus for the fact that overt disease is not developing. It is not specific immunity, because this virus was unknown before December 2019, and more important, unknown to the body's immune defenses. There is no specific immunity to this virus simply because it's new: thus we're talking nonspecific immunity, the so-called natural or innate immunity we all have. Its protective power varies among individuals and depends on several factors.

Understanding how this innate immunity functions in COVID-19 could help us alter its clinical course, reducing the number of people who progress to serious illness. It's a major scientific challenge: to identify and then administer whatever factor that has successfully promoted innate immunity in asymptomatic people. This is a very interesting strategy that can change the outcome of the battle between virus and host, so that individual defenses triumph, boosted by medications that reinforce or enhance innate immunity to control the disease.

MEDICC Review: The biopharmaceutical industry has been playing a key role in Cuba's response to COVID-19. Could you give us an overview of projects under way to address the disease?

Eduardo Martínez: BioCubaFarma is the umbrella corporation for Cuba's pharmaceutical and biotech industry and research institutes. Its institutions are working in four areas related to COVID-19: provision of products approved by the Ministry of Public Health (MINSAP) for the disease; research and development into new knowledge and products; international cooperation to provide medications to help fight the pandemic; and internal measures in our firms to protect workers and ensure operational continuity under current circumstances. We have 25 products, including generic medications (among them chemical antiviral agents) and novel products developed by Cuban researchers.

BioCubaFarma companies have taken on production of masks and sanitizing products as well, and our factories are repairing devices needed to address the pandemic, such as ventilators, and producing personal protective equipment (mainly filter masks, face shields, goggles and gowns). 


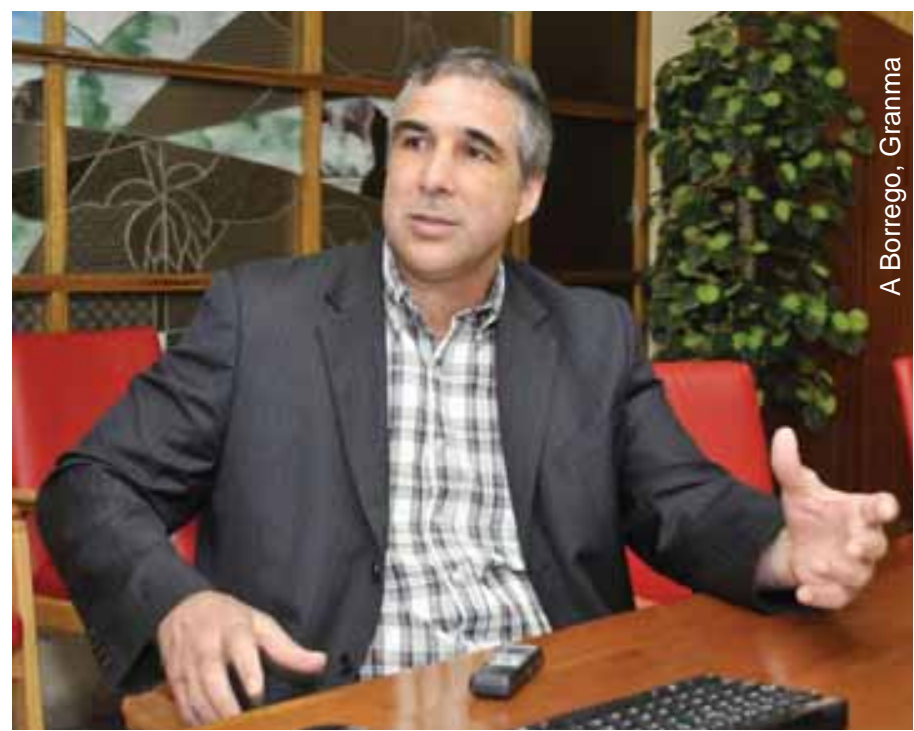

Dr Eduardo Martínez: BioCubaFarma institutions are working in four areas related to COVID-19.

Rolando Pérez: BioCubaFarma is working on 15 projects for COVID-19. Six are treatments, six related to prevention in at-risk groups, two are diagnostics, and one is a medical device. Nine are undergoing clinical trials in patients and at-risk groups, while six are in R\&D. There are also other projects in the design phase or early research stages.

Building on knowledge generated in other countries, we have designed clinical trials and population interventions with products that are already registered or that are in very advanced research stages. All clinical trials and population interventions have been approved by the Center for State Control of Medicines, Equipment and Medical Devices (CECMED), Cuba's regulatory authority, in accordance with ethical principles for conducting clinical trials; their results will be shared with the international scientific community.

We are using interferon alfa-2b, a foundational product of Cuban biotech, which has direct antiviral activity and is an immunostimulant. It is produced by the Genetic Engineering and Biotechnology Center (CIGB) and the National Center for Bioproducts. A nasal formulation is under development. We are also researching the combination of alpha and gamma interferons.

Two products are being evaluated for possible management of hyperinflammatory reactions (the cytokine storm). One is a monoclonal antibody from the Molecular Immunology Center (CIM), which inhibits the effector phase of immunity; the other is a CIGB product, CIGB 258 , which stimulates the immune system's regulatory cells. Both are also in development for treatment of autoimmune diseases.

\section{MEDICC Review: What preventive measures are under devel- opment?}

Rolando Pérez: For prophylactic use in at-risk groups and vulnerable people, various immune system stimulants are being evaluated: - Nasal administration of interferon alfa-2b, a CIGB project in collaboration with AICA Laboratories, a Cuban pharmaceutical company;
- Biomodulin T (produced by Cuba's National Center for Bioproducts), a natural product obtained from thyme extracts, which has been used for several years as a cellular immunostimulant in people aged 60 years and older who experience immunosenescence;

- A transfer factor produced by CIGB from natural sources, which is also a cellular immunostimulant and has been used for more than 30 years in patients with immune deficiencies;

- A new immune potentiator, CIGB 2020, now in clinical trials; and

- VME-corona, from the Finlay Vaccine Institute, a vaccine with certain components of the Cuban vaccine VA-MENGOC-BC, which has been used since the 1980s to control serogroup B meningococcal disease. In addition to its specific properties, this vaccine activates innate immunity. The regulatory authorities approved a study of people at epidemiologic risk, which should start in the next few days.

\section{MEDICC Review: What about disease diagnostics?}

Rolando Pérez: The Immunoassay Center is developing reagents for serologic diagnosis, to detect IgM and IgG antibodies in people who have been infected with the virus. It's an ELISA system, based on Cuba's ultramicroanalytic system (SUMA), and could be available in the next few weeks. Using bioinformatic methods, we've designed peptides characteristic of the SARS-CoV-2 viral structure that can be used to develop ELISA systems for detecting antibodies that recognize the virus. This would enable national coverage, since there are already 3000 SUMA equipment in Cuban health institutions. This is a strength of our national diagnostics system, which could make major contributions to COVID-19 control through expanded detection of infected individuals. Last but not least, several companies in BioCubaFarma, including Cuba's Neurosciences Center and Combiomed (which produces electromedical equipment), are repairing ventilators and designing a new ventilator model, using 3D printing of some parts.

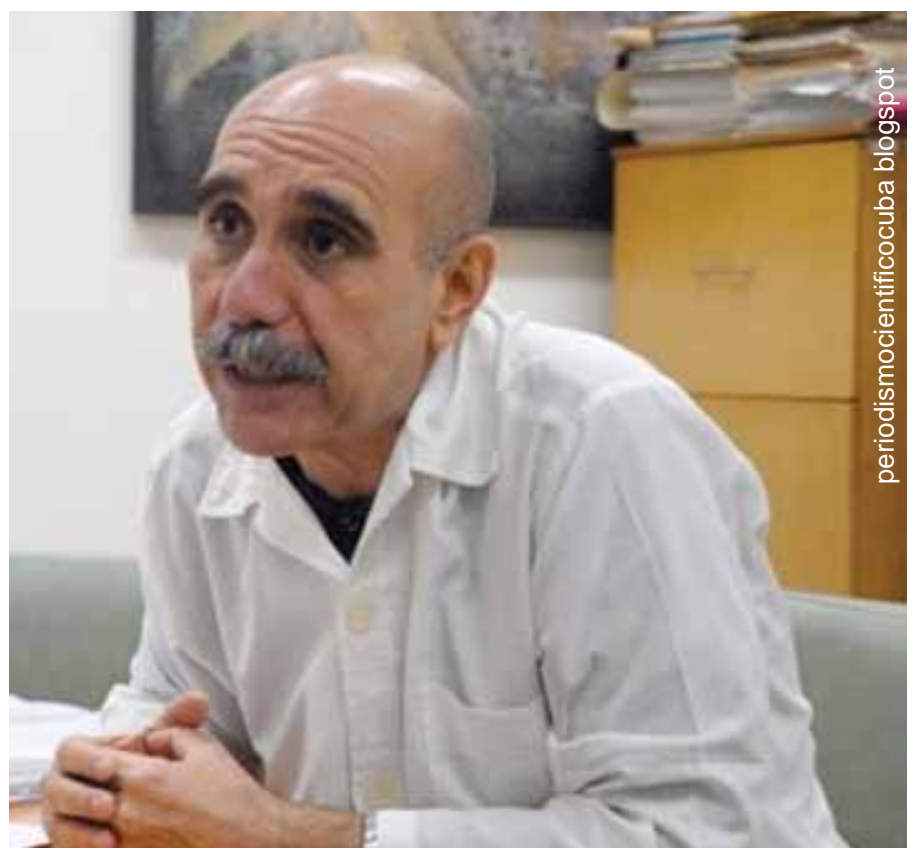

Dr Rolando Pérez: Various immune system stimulants are being evaluated. 
Eduardo Martínez: Expanding detection is key because of course, the asymptomatic carriers represent the biggest threat in terms of disease spread. It's a challenge internationally and here in Cuba. We continue to incorporate more labs for polymerase chain reaction (PCR) diagnostics, but we need a test that we can run on 5000-6000 or more people per day, such as those based on antibodies. These enable detection of asymptomatic carriers and people who have had contact with but are clear of the virus. This is crucial information, because it enables contact tracing. Finding antibodies means that the person has been infected, even when PCR is negative because the virus has been cleared from the body. That's another advantage of an antibody test.

\section{MEDICC Review: What role does interferon play in treating COVID-19?}

Eduardo Martínez: Interferon is produced by the body and has antiviral action, activating virus-inhibiting mechanisms. The SARS-CoV-2 virus reduces interferon levels in the host organism, depriving it of natural antiviral protection. The problem is seen most strikingly in patients who are already immunocompromised and thus particularly vulnerable.

Luis Herrera: Middle East Respiratory Syndrome (MERS), a respiratory disease that appeared in 2012, was caused by a coronavirus that infects humans, bats and camels. That virus invades nonciliated epithelial cells in the bronchi, evades the innate immune system and impedes interferon production by these cells. This led us to propose use of interferon from the beginning of the COVID-19 epidemic in China. Based on those results, expert consensus recommended use of interferon in China and other countries in their treatment protocols (together with chemical antivirals). Its use in Cuba has helped us keep patients from progressing to more dangerous phases of COVID-19.

MEDICC Review: Some media have commented that interferon's effectiveness hasn't been demonstrated in this pandemic. Are there any results yet in Cuba that allow us to assess its effect?

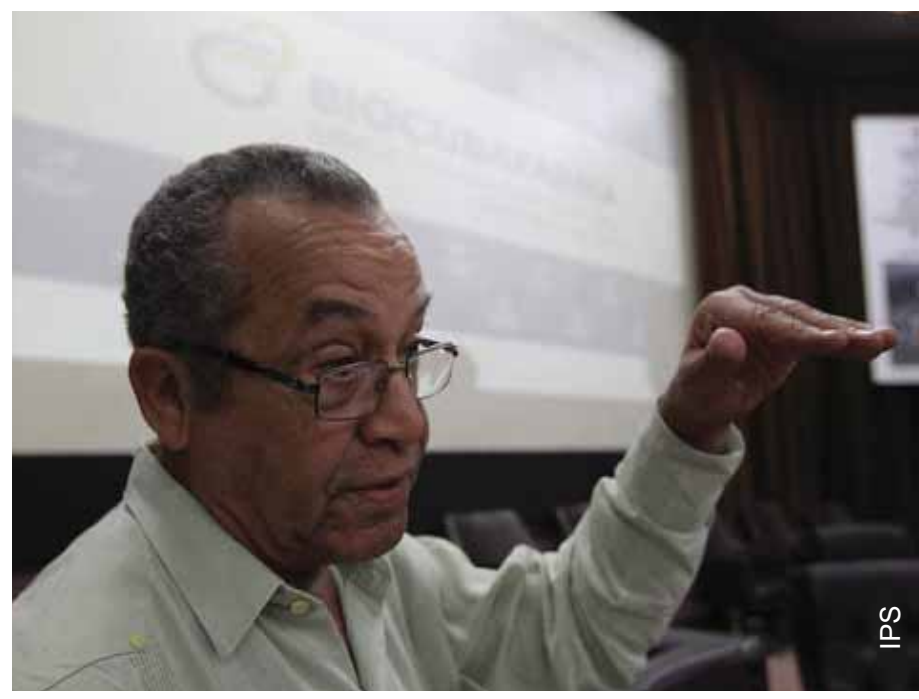

Dr Luis Herrera: Interferon use in Cuba has helped keep patients from progressing to more dangerous phases.

Eduardo Martínez: Not definitively, nor for any antivirals, because this is a novel virus, unknown before December 2019. Thus, there hasn't been time to conduct and obtain results from clinical trials. In fact, WHO has proposed the SOLIDARITY study, running three clinical trials to assess the efficacy of various antivirals and therapeutic approaches used thus far. However, previous experience suggests interferons could be helpful. Interferon application in Cuba is showing benefits, and we plan to publish our results once they are definitive.

MEDICC Review: CIGB 258 is already in use. What is it and how was it developed?

María del Carmen Domínguez: For 20 years now, I have been leading a project to seek new pharmaceutical treatments for chronic respiratory and autoimmune diseases. In these diseases, the immune system gets out of control and produces an inflammatory response against its own molecules, leading to serious health problems.

We now have a number of therapeutic candidates we developed through bioinformatics. Among those, we selected a molecule with a role in regulation of innate immunity and so-called acquired immune memory. Starting with that molecule, we designed new molecules able to regulate the inflammatory response. This involved a broad preclinical research program including assessment of the mechanism of action, toxicology and safety studies. Among the candidates is the molecule named 258. It regulates innate immunity and also induces immune system cells to control inflammation. One of its important characteristics is that it does not induce immune suppression.

Its application in certain COVID-19 patients was approved in turn by the Scientific Councils of CIGB and BioCubaFarma, then by MINSAP's Expert Commission, which approved it for compassionate use (that is, for use in critically ill patients for whom other therapeutic options have been exhausted). Following ethical norms, the next of kin is required to give written consent for use, after receiving a thorough explanation and a document describing in detail the nature of the treatment and its possible benefits and risks. In most cases where CIGB 258 has been applied, C-reactive protein, ferritin and cytokine levels have markedly moved toward normal, and patients are recovering from leukopenia and thrombocytopenia, with substantial improvement in lung imaging.

\section{We are in an emergency and must respond to it, but our response carries with it an important scientific ethical obligation}

I also want to note: we are in an emergency and must respond to it, yes, but our response carries with it an important scientific ethical obligation. Cuban researchers don't see the patient as a client, but as a human being affected by a complex disease and who needs our help. When we act, we do so respecting rigorous ethical standards in the midst of the eagerness we all feel to respond. We're not talking about a cure; we're talking about treatment options. It's crucial to keep patients from reaching the point where they need this molecule. That's why I emphasize the importance of taking and maintaining all the preventive, protective measures advised by health authorities. 


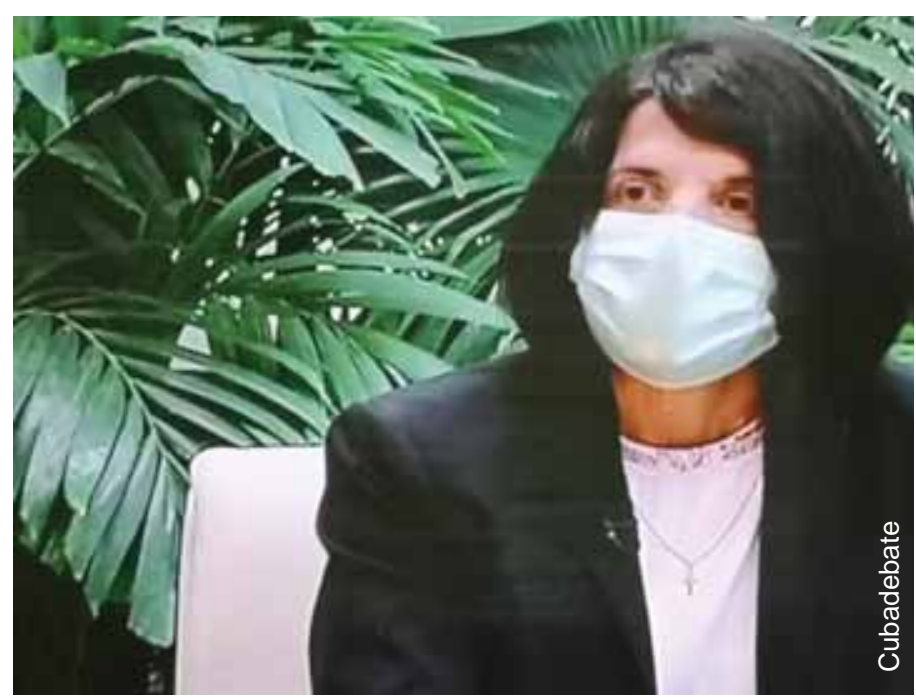

Dr María del Carmen Domínguez, leading projects for two decades on new pharmaceutical treatments for chronic respiratory and autoimmune diseases.

\section{MEDICC Review: What is Cuba's experience in therapeutic} use of vaccines?

Vicente Vérez: In Cuba we have two vaccines that are powerful stimulants of innate immunity, one developed by the Finlay Vaccine Institute, called VME-corona, and one by CIGB, known as CIGB 2020. I'll say a bit more about the first. In 1980, Cuba suffered a meningitis $B$ epidemic that cost many lives, mainly those of young people. Finlay Vaccine Institute researchers under the leadership of Dr Concepción Campa developed VAMENGOC-BC, the world's first vaccine for preventing serotype $B$ meningococcal disease. Mass administration allowed us to put an end to the epidemic.

For nearly 40 years, now, Cuban scientists have been studying the biologic mechanisms that explain VA-MENGOC-BC's efficacy. Their research has shown that certain of its components are efficient potentiators of the innate immune system. As a result, those components have also been used for years in some of our novel therapeutic vaccines against cancer developed by $\mathrm{CIM}$ and in vaccines against allergies. Cancer and allergies have nothing to do with the original protection target-meningococcal diseasebut components of the vaccine are able to stimulate the innate immune system. Since we had so many years of research on the mechanisms for stimulating innate immunity by a product, which moreover had been shown to be safe and is in wide use in Cuba and several other countries, we considered the possibility that it might be helpful to address the current pandemic.

Gerardo Guillén: At CIGB, we're working on a vaccine called CIGB 2020. It's administered nasally or sublingually and stimulates innate immunity locally, precisely along the route of infection for a respiratory disease. We have a trial under way with volunteers, showing preliminary evidence of innate immune system activation. Specifically, the vaccine activates cell surface receptors in the nasopharyngeal mucosa that recognize repetitive patterns as in viral RNA. Systemically, you can detect stimulation of lymphocytes and macrophages in blood; these present viral antigens to the immune system and thus stimulate a specific response to the coronavirus. In sum, these studies are showing innate immunity activation and preparation of the immune system to develop specific immunity against the virus. The study began recently; when it is complete and we assess the product in a larger group of volunteers, we'll know to what extent this response brings about effective antiviral protection.

This research doesn't involve only CIGB, but was also made possible thanks to cooperation by MINSAP, which approved the study in record time, and to participation by specialists at Havana's Luis Díaz Soto Naval Hospital, whose ethics committee also approved the trial. On March 26, 2020, the clinical trial was approved with participation of an expert group from MINSAP, the National Clinical Trials Coordinating Center (CENCEC) and CECMED. On March 27, a workshop was held to initiate the study involving medical and laboratory personnel, clinical researchers and hospital directors. Volunteer recruitment began among people with symptoms (suspected cases) or contacts of confirmed cases. Volunteers were informed in detail about the trial and provided written consent, according to ethical norms for such clinical trials.

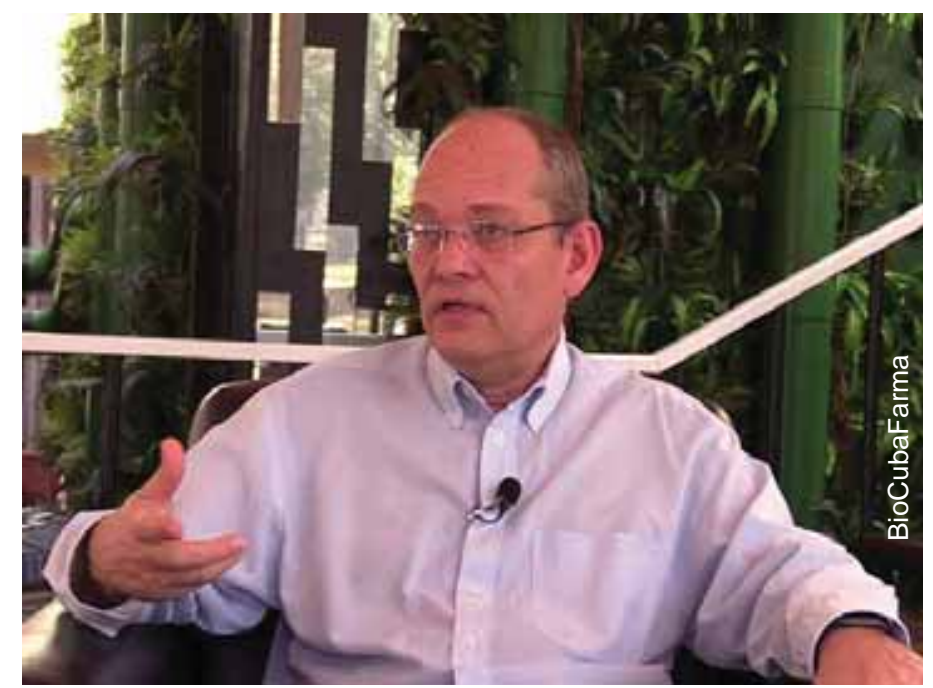

Dr Gerardo Guillén: Limited studies of CIGB 2020 are showing innate immunity activation and preparation of the immune system to develop specific immunity against the virus.

Vicente Vérez: Turning to VME-corona, we've launched a collaborative project to evaluate it with the Pedro Kourí Tropical Medicine Institute (IPK), MINSAP authorities and hospitals assigned to manage coronavirus cases. We aim to determine what benefits we can derive from this vaccine, whose biology and immunology we know well, if we include it in treatment approaches to reduce the number of asymptomatics who become ill and reduce the number of patients who become seriously ill.

The project extends to apparently healthy people, in whom the product might have a nonspecific preventive effect. I emphasize that this is nonspecific, acting on the innate immune system; it is not a specific vaccine. In the first phase, it will be given to particular at-risk groups. We're still finalizing logistics, because administering vaccines is complicated right now with social distancing. All actions will be explained to the community; we'll inform people in detail of what we plan to do and how our approach is based on almost 40 years of research. All participants will be given detailed 
information about the trial and its possible risks and benefits and will need to provide written consent. All results will be shared with the international scientific community.

MEDICC Review: Is Cuba looking for a specific vaccine against SARS-CoV-2, as others are doing?

Vicente Vérez: The Finlay Vaccine Institute has been working intensively on a vaccine against pneumococcus, a priority for protecting our children. We still haven't eliminated pneumococcal pneumonia in Cuba. Nonetheless, we've now paused that research to tackle this huge problem; when this emergency has passed, we'll continue the pneumococcal vaccine work until we have this vaccine for our national health system. Developing a vaccine against SARS-CoV-2 will need highly integrated efforts by our entire research and industrial capacity. CIGB is working on the virus's specific antigens, which will provide a platform for the projects, while CIM has considerable experience using mammalian cells for biotech production.

The Finlay Vaccine Institute has undertaken three projects to explore and understand the way the virus functions. The virus has many evasion mechanisms and designing a vaccine is impossible without understanding them. One of the most important is an expansion mechanism first observed in HIV in 2000. The virus sequesters immune system cells and puts them to work to help it multiply. In 2004, this phenomenon was also seen in the first SARS virus (SARS-CoV-1), the closest cousin to this new coronavirus. When that SARS virus enters immune system cells, it forms a double vacuole that hides many of the molecular characteristics that normally enable the immune system to identify and attack it. This viral expansion is infrequent. Might SARS-CoV-2 also be using this mechanism? How might we counter it? So we're not just looking for antigens specific to this virus; we're trying to understand the tricks it uses to its advantage. Once we understand these mechanisms, we'll be able to develop a swifter strategy to design both vaccines and treatments.

Gerardo Guillén: A specific vaccine is a tremendous challenge. Some pandemics have disappeared even without a vaccine being discovered in time. There's no evidence yet to suggest that risk of viral contagion will disappear within a few months, so many groups worldwide are working on a preventive vaccine. Because of containment measures to protect the population and prevent deaths, much of the world's population will not have had contact with the virus, won't have developed immunity and will continue to be susceptible as long as the virus continues to circulate. That's why specific vaccines are needed. A specific preventive vaccine could take years to develop. The most optimistic scientists speak in terms of a year to 18 months of intense development.

Cuban institutions working toward such a vaccine are designing various strategies, beginning with selection of viral antigen fragments and their expression in mammalian cells, bacteria and yeast. Such cell systems are used in biotech to produce proteins for use in both treatments and vaccines. After this first step, we'll have to develop purification processes, analytic systems, and in vitro and in vivo studies. These projects require participation from several areas of our institutions, including research, product development, animal research, toxicology and pharmacology. Finally, only candidates that successfully pass through this entire trajectory proceed to clinical trials to assess their safety and efficacy in humans.
MEDICC Review: How important is international collaboration in this research?

Eduardo Martínez: Cuba is willing to collaborate on research and other areas as well, sharing experiences. A number of countries have also requested Cuban cooperation, not just with health personnel, but also with medications. For example, 72 countries have asked for interferon. We have a production plant in China and one here. We also have factories that make other medications, which we could convert to interferon production if needed at some point. Finally, of course, Cuban institutions are collaborating in every way possible with $\mathrm{WHO}$ and $\mathrm{PAHO}$, indispensable for any effective global response to COVID-19.

Gerardo Guillén: International collaboration is essential to move ahead with development of specific vaccines. It's highly unlikely that any single institution would have all the methodology, equipment, animal models and financial resources to carry out a project of this magnitude quickly and successfully. For example, animal studies require exposing animals to the virus. There are reports of this kind of research on coronavirus using transgenic mice, ferrets and monkeys. This has to be done in highly secure Level-3 laboratories. We have links with Chinese colleagues who have such conditions for experimental research. Only joint efforts through these collaborations will carry us through all the essential stages of this complex research program.

MEDICC Review: Cuba has collaborated with China on biotech production for years such as the joint ventures Biotech Pharmaceuticals, to produce monoclonal antibodies against cancer, and ChangHeber for interferon alfa-2b production, the first with CIM technology, the second with CIGB technology. Can you describe Cuba's collaboration with China concerning COVID-19?

Eduardo Martínez: We have a group of scientists working in China. Last year, we created a Chinese-Cuban R\&D center. Information from our representatives in China has been systematically compiled over the course of the pandemic and has been very important. Much of it we received before it appeared in the mass media and helped us form an idea of what was going on and what our contribution could be.

Luis Herrera: The Chinese-Cuban joint venture, ChangHeber, was created in 2003, with headquarters in the city of Changchun, to produce interferon alfa- $2 \mathrm{~b}$. As we mentioned, this product is being used to treat COVID-19 in China, selected by the country's State Health Commission. ChangHeber, together with Biotech Pharmaceuticals and Changchun Heber Biological Technology, are the results of Chinese-Cuban collaboration in biotech, a sector with ample prospects for joint projects.

MEDICC Review: Antibacterial chemotherapy has had great success, despite the phenomenon of resistance. In the field of antivirals, with few exceptions (such as acyclovir), it was only with the onset of the AIDS epidemic that concerted research was directed at antiviral chemotherapy. In fact, what stopped AIDS from spreading was not discovery of a vaccine (still pending), but the success of antivirals. How is research on antivirals proceeding globally, and what can we expect in the next few years? 


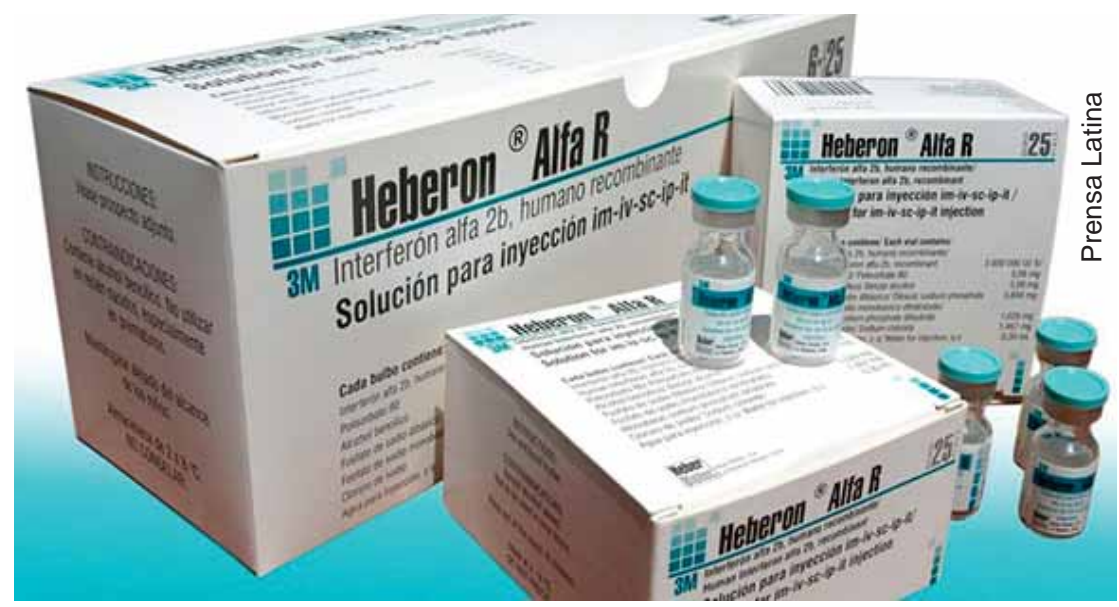

Cuban biotech's interferon alfa-2b is being used in China and other countries as an antiviral and immune booster during the COVID-19 pandemic.

Luis Herrera: Drug repurposing or drug reprofiling is an established practice in the pharmaceutical industry. Accumulated knowledge of how a drug acts provides a precedent for trying it for other diseases; based on that knowledge, we form hypotheses about its possible action against a new disease. This increases the chances of finding a successful drug. The previously designed drug has already passed all the required steps to obtain regulatory approval, especially in regard to safety, production requirements and molecular structure. So there's no need to repeat all those expensive and time-consuming steps. Several drugs already approved for other viral diseases are going into clinical trials to see whether they are effective against this new coronavirus.

MEDICC Review: What can we learn from other recent epidemics to help us deal with the COVID-19 pandemic?

Vicente Vérez: We can't wait to develop a pharmaceutical product from zero, which takes a long time, because the pandemic won't let us. We all dream of a preventive vaccine that can resolve the problem, but unfortunately that solution isn't within our grasp this year, no matter how hard the international scientific community works. Because, additionally, most of the technologies needed are new themselves. Until we have such a preventive vaccine, we have to put up barriers against the virus. Today the best "vaccine" is prevention via physical distancing. We have to learn from history: the SARS epidemic ended before a vaccine could be developed. The same thing happened with Ebola. The world is facing a critical juncture: What will happen epidemiologically when countries emerge from the current epidemic phase and reduce social isolation restrictions, returning to "normal" life? We don't know whether the epidemic will end, as others have, or if the virus has come to stay.

We are working passionately. It's a very complex problem that scientists the world over are tackling. Here in Cuba, we hope to apply what we've learned about the innate immune system and administer products that are the fruit of years of research and massive use, taking advantage of this considerable experience and accumulated knowledge, particularly about their immunological mechanisms of action. Such products can help address urgent needs, before finding a preventive vaccine. This is the path we have to pursue with all our intelligence, a battle we have to win.

Eduardo Martínez: One important lesson is that most countries lacking a solid pharmaceutical industry have a serious problem: scarcity of drugs used for treatment and inability to meet demands to tackle the pandemic. Cuba can count on domestic production of antiviral medications, particularly interferon alfa-2b, and that gives us an advantage going forward.

Editorial note: This roundtable is the result of original MEDICC Review telephone and email interviews with participants complemented by published comments from them as necessary for clarification. -1 - 